Australian

\section{VETERINARY JOURNAL}

C L I N I C A L S E C T I O N
EDITOR
MAUREEN REVINGTON
ADVISORY COMMITTEE
ANAESTHESIA
LEN CULLEN
AVIAN MEDICINE AND SURGERY
GARRY CROSS
EQUINE MEDICINE AND SURGERY
JOHN BOLTON
LABORATORY ANIMAL MEDICINE
MALCOLM FRANCE
OPHTHALMOLOGY
JEFF SMITH
PATHOLOGY
CLIVE HUXTABLE
PHARMACOLOGY / THERAPEUTICS
STEPHEN PAGE
PRODUCTION ANIMAL MEDICINE
JAKOB MALMO
RADIOLOGY
ROBERT NICOLL
REPRODUCTION
PHILIP THOMAS
SMALL ANIMAL MEDICINE
BRIAN FARROW
DAVID WATSON
SMALL ANIMAL SURGERY
GEOFF ROBINS
GERALDINE HUNT
WILDLIFE / EXOTIC ANIMALS
LARRY VOGELNEST
JOURNAL ABSTRACTS
MAUREEN REVINGTON
EDITORIAL ASSISTANT AND
DESKTOP PUBLISHING
ANNA GALLO
M

CONTRIBUTIONS INVITED

Practising veterinarians and others are invited to contribute clinical articles to the Australian Veterinary Journal. We will consider material in a variety of formats, including clinically orientated reviews, reports of case series, individual case studies, diagnostic exercises, and letters containing comments or queries. Contributors should consult instructions for authors on http://www.ava.com.au/avjintro and recent issues of the journal for guidelines as to formatting

Over referencing should be avoided: authors should preferably quote only those articles they feel are most likely to be of interest and benefit to readers.

Send all contributions to:

Editor, AVJ Clinical Section

AVA House, 272 Brunswick Road,

Brunswick, Vic 3056

Phone: (03) 93872982

Fax: (03) 93880112

Email: desktop@ava.com.au

Authors, please note that from July 1st 2005, the manuscript handling fee will be $\$ 55$, including GST.

\section{Clinicopathological findings associated with feline infectious peritonitis in Sydney, Australia: 42 cases (1990-2002)}

\author{
JM NORRIS ${ }^{\text {ad }}$, KL BOSWARD ${ }^{\mathrm{a}}$, JD WHITE ${ }^{\mathrm{a}}, \mathrm{RM} \mathrm{BARAL}^{\mathrm{b}}$, MJ CATT $^{\mathrm{b}}, \mathrm{R}^{\mathrm{B}}$ MALIK $^{\mathrm{c}}$
}

Objectives To review the clinicopathological findings in naturally-occurring, histopathologically confirmed cases of feline infectious peritonitis in client-owned cats in Sydney, Australia, with the purpose of identifying factors assisting in the diagnosis of this complex disease syndrome and to characterise the disease as it occurs in this region.

Design Retrospective clinical study: the clinical records of all cats with histopathologically confirmed feline infectious peritonitis at the University Veterinary Centre Sydney and a private cat hospital in Sydney between 1990 and 2002 were reviewed for signalment, history, physical findings, diagnostic test results and the distribution of histological lesions throughout the body at necropsy.

Results Forty-two cats met the inclusion criteria. Significant features of this study that unique to the contemporary literature are i) the over-representation of certain breeds (Burmese, Australian Mist, British Shorthaired, and Cornish Rex) and the under-representation of other breeds (Domestic Shorthaired, Persian); ii) the overrepresentation of males; iii) the tendency for effusive disease in Australian Mist cats and non-effusive disease in Burmese; iv) the even age distribution of disease seen in cats older than 2 years-of-age; and v) the presence of fulminant immune-mediated haemolytic anaemia in two cats in this study.

Conclusion The study highlights the diverse range of clinical manifestations and the complexities experienced by clinicians in diagnosing this fatal disease. Some aspects of the epidemiology and clinical manifestations of feline infectious peritonitis appear different to the disease encountered in Europe and North America, most notably the over-representation of specific breeds and the presence of immune-mediated haemolytic anaemia.

Aust Vet J 2005;83:666-673

\begin{tabular}{ll} 
ALT & Alanine aminotransferase \\
ALP & Alkaline phosphatase \\
AST & Aspartate aminotransferase \\
BSH & British Shorthaired cats \\
CK & Creatine kinase \\
CNS & Central nervous system \\
CSF & Cerebrospinal fluid \\
DSH & Domestic shorthaired cats \\
FCoV & Feline coronaviruses \\
FECV & Feline enteric coronavirus \\
FIP & Feline infectious peritonitis \\
FIPV & Feline infectious peritonitis virus \\
FNA & Fine needle aspirates \\
GIT & Gastrointestinal tract \\
LCAT & Latex Cryptococcal Agglutination Test \\
LDV & Lactate dehydrogenase-elevating virus \\
PCH & Paddington Cat Hospital, Sydney \\
RR & Reference range \\
RT-PCR & Reverse transcriptase polymerase chain reaction \\
UVCS & University Veterinary Centre Sydney \\
\hline
\end{tabular}

${ }^{a}$ Faculty of Veterinary Science, Building B14, The University of Sydney, New South Wales, 2006

baddington Cat Hospital, 183 Glenmore Road, Paddington, New South Wales, 2021

${ }^{\mathrm{C}}$ Post Graduate Foundation in Veterinary Science, Veterinary Conference Centre, B22, The University of Sydney, New South Wales, 2006

dCorresponding author jnorris@mail.usyd.edu.au 
IP is a fatal immunopathological disease of wild and domesticated Felidae caused by mutant variants of the ubiquitous feline enteric coronavirus, known as feline infectious peritonitis virus. ${ }^{1,2}$ The disease was first described in 1963 by Holzworth $^{3}$ as a syndrome known as 'chronic fibrinous peritonitis', but is now characterised by varying degrees of systemic serositis, vasculitis and disseminated pyogranulomas. The first reported appearance of FIP in Australia was in $1974^{4,5}$ but despite the anecdotal evidence of its frequent occurrence in Australia, there has been a conspicuous absence of peer-reviewed reports since that time.

Viruses within the family Coronaviridae, like many other RNA viruses, are known for their tendency to mutate frequently during replication. Feline coronaviruses $(\mathrm{FCoV})$ are typical in this regard. Although attention has focused on the group specific gene clusters $3 \mathrm{abc}$ and $7 \mathrm{ab}$ of FCoV in an attempt to discover factors that confer greater virulence to certain strains, the exact nature of the FIP inducing mutations are still unknown. 1,2,6,7 To complicate matters further, the role of the immune system in protecting against disease in some animals, while contributing to the disease in others, is another poorly defined phenomenon in the pathogenesis of FIP. Cats with a competent immune system infected with less pathogenic strains of FIPV may avoid overt disease manifestations, however genetic susceptibility or the immunomodulating effects of stress or concurrent disease may allow the development of clinical disease with less virulent strains. $^{2}$

The clinical presentations of FIP are variable and often complex, reflecting variations in the virus and the nature of the host immune response. Approximately 50\% of all cats diagnosed worldwide are less than 2-years-old and purebred cats are generally over represented. Two broad forms of the disease have been described across all ages and breeds: 'effusive' and 'non-effusive'. Despite this apparent division, these are not distinct disease entities. Cats with FIP may alternate between forms during the progression of disease. Furthermore, cats with non-effusive FIP may develop effusions in the terminal stages of disease, and conversely, there are reports of non-effusive FIP being preceded by a subtle effusive form. ${ }^{8}$ Typically, patients with effusive FIP have high protein peritoneal and/or thoracic effusion(s), fever, weight loss, anaemia and elevated serum globulin levels, although not all cats adhere to this stereotype. The non-effusive form of FIP is often more vague in its presentation with nonspecific signs including fever, weight loss, malaise and inappetance. Clinical signs, beyond the non-specific, relate to the tissues affected. Possibilities include liver, kidney, pancreas, spleen, abdominal lymph nodes, CNS, lung, GIT, eyes and heart. Usually more than one body system is involved but occasionally only one is affected, most frequently the CNS or GIT, and clinical signs are restricted to this particular system.

The diagnosis of FIP is a complicated and controversial issue. It has been described as one of the most over diagnosed and misdiagnosed diseases of cats due to its complex and variable pathogenesis and the over reliance on serology for diagnosis. ${ }^{9}$ Clinicians confronted with a very sick and, frequently, young cat with clinicopathological findings suggestive of FIP often find it difficult to confirm the diagnosis definitively using non-invasive methods. RT-PCR on blood, effusions or tissue (not available in Australia) and serum antibody tests have been considered by some to be helpful in supporting a clinical diagnosis of FIP, but they must be considered together with the patient's history and other clinico- pathological findings and can never be used alone. No peer reviewed scientific data has been published outlining the pathogenic role of mutated genes and proteins detectable by RT-PCR or serology. ${ }^{10}$ Of greater concern is the finding by several researchers of purported FIPV infection in healthy asymptomatic cats, ${ }^{11,12}$ highlighting the need to exercise care with interpretation. Recent developments in the detection of replicating FCoV within circulating monocytes by RT-PCR look more promising. ${ }^{13}$ The detection of characteristic histopathological lesions has been considered the only conclusive test for FIP for a considerable time $^{14}$ and the finding of viral antigen within macrophages in the lesions using immunohistochemistry allows definitive confirmation of the diagnosis. In a disease process where the treatment of choice is often euthanasia, diagnostic accuracy is essential.

The objectives of this study were to compare the clinical manifestations of FIP in part of Australia's geographically isolated cat population with overseas reports and to identify risk factors and clinical and laboratory findings that may aid in the diagnosis of this complex disease.

\section{Materials and methods}

Selection of cases

Clinical pathology and histopathology records from Veterinary Pathology Diagnostic Services, The University of Sydney, between 1990 and 2002, were reviewed to identify cats diagnosed with FIP. This laboratory processes clinical samples from the UVCS (primary accession and referral clinic) and PCH (primary accession clinic). Only cats in which the diagnosis was confirmed histopathologically as the result of necropsy or biopsy were included.

\section{Data collection}

The medical records of confirmed cases were reviewed. Data concerning the cat's age, breed and gender, as well as the history, physical findings, results of diagnostic tests performed, and the distribution of histopathological lesions throughout the body, were recorded.

\section{Analysis of data}

All statistical analyses were performed using two-tailed Fisher's exact tests (www.matforsk.no/ola/fisher.htm) by constructing two-by-two contingency tables. Comparisons were made between the cohort of cats with FIP and other cats presented to the UVCS and $\mathrm{PCH}$ over the same period.

\section{Results}

Cases

The case records of 42 cats with histopathologically confirmed FIP from the UVCS (32 cats) and PCH (10 cats) patient databases between 1990 and 2002 were reviewed. Full necropsies had been performed in 37 cats, while five had been diagnosed by biopsy. Necropsies are routinely offered free of charge at both hospitals, accounting for the large proportion of cases in which this was performed.

\section{Signalment}

Age - Of the 42 cats with FIP, 22 (55\%) were less than 2-yearsold, while $20(45 \%)$ were between 2- and 11-years-old, with an even distribution of ages in this latter group. Cats less than 2 years-of-age were significantly over-represented in this disease group compared with the hospital population: this age group normally accounts for $21 \%$ of non-FIP cases. The age of cats at diagnosis is presented in Figure 1. 


\section{Number of cats in each age group at the time of diagnosis}

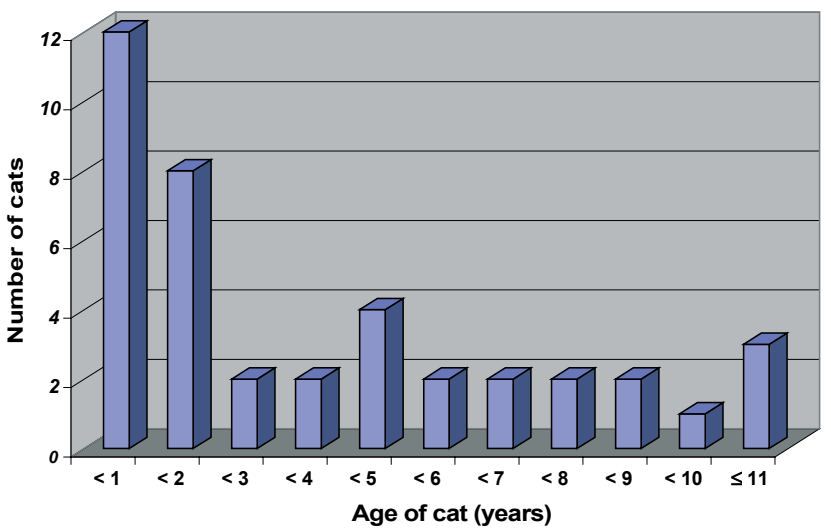

Figure 1. Number of cats in each age group diagnosed with feline infectious peritonitis in a study of 42 histopathologically confirmed cases.

Breed - Pedigree cats accounted for 30/42 (71\%) of the cohort, while 12/42 (29\%) were DSH. The number of cats diagnosed with FIP in each breed were as follows: DSH (12), Burmese (10), Australian Mist (5), Siamese/Oriental/Balinese (5), BSH (3), Birman (2), Cornish Rex (2), Ragdoll (1), Abyssinian (1), and Exotic (1). Overall, purebred cats were over-represented when compared to the hospital population $(\mathrm{P}<0.01)$. Burmese, Australian Mist, BSH and Cornish Rex cats were over represented $(\mathrm{P}<0.01)$, while DSH $(\mathrm{P}<0.01)$ and Persians $(\mathrm{P}<0.05)$ were under-represented when compared to the hospital population.

Gender - Twenty-seven of $42(64 \%)$ cats with FIP were male while $15(36 \%)$ were female. Males were significantly over-represented when compared to the hospital population $(\mathrm{P}<0.05)$. Most breeds within this study had too few numbers to analyse the male: female ratio, however in Burmese cats the ratio of males to females was 7:3

\section{Historical and physical findings}

Findings varied considerably between cats and included combinations of the following: inappetance, weight loss, pale mucous membranes, fever, abdominal distension, vomiting, diarrhoea, jaundice, palpable abdominal organomegaly, multifocal neurological signs, abdominal pain, dyspnoea, uveitis and mandibular lymphadenomegaly (Table 1). One cat presented dead on arrival so only some physical findings could be recorded.

Effusive FIP was found in 20/42 cats (45\%; UVCS 14/32; PCH $6 / 10)$. Non-effusive FIP was diagnosed in $22 / 42$ cats $(55 \%$; UVCS 18/32; PCH 4/10). Of the 32 cases of FIP seen at UVCS, 20 were referrals or second opinions; 11 of these cats had noneffusive disease, while nine were effusive. Equal numbers of effusive and non-effusive cases were seen as primary accession consults. No statistical relationship was found between the type of disease seen and whether the case was a referral or seen as a primary consult.

Equal numbers of DSH were seen with effusive and non-effusive disease. Of the 10 Burmese cats with FIP, seven had the non-effusive form. Of the five Australian Mist cats with FIP, four had effusive FIP and all were under 2-years-old, while the one cat with non-effusive disease was 4-years-old.
Table 1. Historical or physical findings in 42 cats with feline infectious peritonitis.

\begin{tabular}{ll}
\hline Clinical signs & $\begin{array}{c}\text { Number of cats with } \\
\text { clinical signs (percentage) }\end{array}$ \\
\hline Inappetance & $41(98 \%)$ \\
Weight loss & $36(88 \%)^{\mathrm{a}}$ \\
Pale mucous membranes & $28(68 \%)^{\mathrm{a}}$ \\
Fever & $26(63 \%)^{\mathrm{a}}$ \\
Palpable abdominal organ enlargement & $20(48 \%)^{\mathrm{b}}$ \\
$\quad$ GIT & 15 \\
$\quad$ Abdominal lymph node(s) & 9 \\
Kidney & 3 \\
Gastronded abdomen/ascites & $19(45 \%)$ \\
(diarrhoea and/or vomiting) & $15(36 \%)$ \\
Dyspnoea & \\
Jaundice & $12(29 \%)^{\mathrm{a}}$ \\
Multifocal neurological signs & $11(26 \%)$ \\
(ataxia, central depression, & $10(24 \%)^{\mathrm{a}}$ \\
paresis, seizures and/or hyperaesthesia) & \\
Abdominal pain & \\
Uveitis & \\
Palpable submandibular lymph node enlargement & $6(15 \%)^{\mathrm{a}}$ \\
Polyuria/Polydispia & $5(12 \%)$ \\
\hline
\end{tabular}

aOut of 41 cats only, as one cat was presented dead on arrival and some clinical signs could not be assessed.

bSeven cats had more than one abdominal organ palpably enlarged.

\section{Diagnostic tests}

Haematology — Of the 42 cats with FIP, full haematological analyses were performed in 29; 10 had only PCV determinations, while in three cats no haematological analyses were performed. Of the 29 cats with full blood count analysis, 17 had a non-regenerative anaemia, while four of the 10 cats had a low PCV when this was the only aspect of haematological analysis performed (Table 2 ). Thus $21 / 39$ cats $(54 \%)$ were anaemic.

Two cats $(4.7 \%)$ in which full haematological analyses were performed were presented during a haemolytic crisis. In both cases haemolysis was refractory to treatment. While the aetiology of the haemolysis was not definitely determined in either case, it was considered to be the result of either secondary immune-mediated haemolytic anaemia and/or vasculitis with loss of erythrocytes into the tissue. The presence of erythrophagocytosis by macrophages seen in the mesenteric lymph node biopsy of one of the cats in this study (Figure 2) supported a diagnosis of secondary immune-mediated haemolytic anaemia.

Of the 29 cats in which full haematological analyses were performed, 16 (55\%) had neutrophilic leukocytosis (Table 2). Sixteen cats $(55 \%)$ were lymphopenic, of which four had associated leukopenia. One cat had mild lymphocytosis.

\section{Biochemistry}

Serum biochemistry analyses were performed in 27 cats. The analyses included some or all of the following: CK, ALT, AST, ALP, urea, creatinine, inorganic phosphate, calcium, and glucose. Eleven of 24 cats (46\%) had hyperbilirunaemia with concurrent jaundice. Three of 23 cats (13\%) had increases in serum ALT above the reference range, while 6/7 (86\%) had increases in AST (Table 2). 
Table 2. Abnormal haematological and serum biochemical findings in cats with feline infectious peritonitis.

\begin{tabular}{|c|c|c|c|}
\hline & Number of affected cats & Abnormal range & Normal values \\
\hline \multicolumn{4}{|c|}{ Haematological findings } \\
\hline Hyperproteinaemia $^{a}$ & $18 / 30(60 \%)$ & $80-132 \mathrm{~g} / \mathrm{L}$ & $59-78 \mathrm{~g} / \mathrm{L}$ \\
\hline $\begin{array}{l}\text { Anaemia } \\
\text { Erythrocyte count } \\
\text { PCV }\end{array}$ & $21 / 39(54 \%)$ & $\begin{array}{l}3-5 \times 10^{9} / \mathrm{L} \\
0.16-0.24 \mathrm{~L} / \mathrm{L}\end{array}$ & $\begin{array}{l}6-10 \times 10^{9} / \mathrm{L} \\
0.25-0.45 \mathrm{~L} / \mathrm{L}\end{array}$ \\
\hline Leukocytosis & $16 / 29(55 \%)$ & $14-67 \times 10^{9} / \mathrm{L}$ & $8-14 \times 10^{9} / L$ \\
\hline Leukopenia & $4 / 29(14 \%)$ & $0.2-7.4 \times 10^{9} / \mathrm{L}$ & $8-14 \times 10^{9} / L$ \\
\hline Neutrophilia & $16 / 29(55 \%)$ & $13-52 \times 10^{9} / \mathrm{L}$ & $3.76-10.0 \times 10^{9} / \mathrm{L}$ \\
\hline Lymphopenia & $16 / 29(55 \%)$ & $0.18-1.5 \times 10^{9} / \mathrm{L}$ & $1.6-7.0 \times 10^{9} / \mathrm{L}$ \\
\hline Lymphocytosis & $1 / 29(3 \%)$ & $9.0 \times 10^{9} / \mathrm{L}$ & $1.6-7.0 \times 10^{9} / \mathrm{L}$ \\
\hline \multicolumn{4}{|l|}{ Biochemical findings } \\
\hline Hyperproteinaemia ${ }^{b}$ & $15 / 27(56 \%)$ & $80-119 \mathrm{~g} / \mathrm{L}$ & $54-73 \mathrm{~g} / \mathrm{L}$ \\
\hline Hyperglobulinaemia & $13 / 27(48 \%)$ & $52-105 \mathrm{~g} / \mathrm{L}$ & $26-51 \mathrm{~g} / \mathrm{L}$ \\
\hline Hyperbilirubinaemia & $11 / 24(46 \%)$ & $20-155 \mathrm{mmol} / \mathrm{L}$ & 2.5 - $3.5 \mathrm{mmol} / \mathrm{L}$ \\
\hline Elevated ALT & $3 / 23(13 \%)$ & $85-147 \mathrm{U} / \mathrm{L}$ & $0-80 \mathrm{U} / \mathrm{L}$ \\
\hline Elevated CK & $7 / 18(39 \%)$ & $899-6024$ U/L & $0-200 \mathrm{U} / \mathrm{L}$ \\
\hline Elevated AST & $6 / 7(86 \%)$ & $64-302 \mathrm{U} / \mathrm{L}$ & $0-48 U / L$ \\
\hline
\end{tabular}

$\mathrm{a}_{\text {determined by refractometer; }}{ }^{\mathrm{b}}$ determined by biuret method

$\mathrm{PCV}=$ packed cell volume $; \mathrm{CK}=$ creatine kinase $; \mathrm{ALT}$ = alanine aminotransferase $; \mathrm{AST}=$ aspartate aminotransferase

Total plasma protein measured by refractometry was above the reference range in $18 / 30$ cats $(60 \%)$ in which this was performed. Hyperproteinaemia was present in equal proportions of cats with effusive and non-effusive disease. Fifteen of 27 cats (56\%) had serum protein above the normal range when measured by the biuret method with 13 of these cats having serum globulins increased above the reference range. One cat had hypoproteinaemia $(45 \mathrm{~g} / \mathrm{L}$; RR 55 to $73 \mathrm{~g} / \mathrm{L})$.

\section{Serology}

Serum antibody titres against products of the $7 \mathrm{~B}$ gene (commercially known as the 7B FIP ELISA test) was measured in one cat. This was determined to be $1: 80$, a level not considered to be consistent with FIP, as cats harbouring the virus are usually considered to have titres greater than 1:160. The Coronase ELISA, which determines the titre of antibodies to coronaviruses, was performed on one cat. This was determined to be 1:800. Levels greater than 1:400 are considered suspicious of FIP but are unable to differentiate between different coronavirus strains. In four cats diagnosed with FIP between 1990 and 1998, the presence of antibodies against coronavirus was determined by competitive ELISA (CELISA, Diasystems ${ }^{\circledR}$ ). Three of the four cats were positive for coronavirus antibodies while one cat was negative.

\section{Abdominal or pleural fluid analysis}

Of the 20 cats with effusive FIP, the complete fluid analysis results (protein, nucleated cell counts and cytological examination via cytocentrifugation) were available in the case records of 10 cats, while a further three had protein determinations by refractometry (Table 3). The protein level of the effusions ranged from 30 to 78 $\mathrm{g} / \mathrm{L}$ (median $57 \mathrm{~g} / \mathrm{L}$ ) with 9 of the 10 cats greater than $50 \mathrm{~g} / \mathrm{L}$. One cat with an effusion protein level of $30 \mathrm{~g} / \mathrm{L}$ also had mild serum hypoproteinaemia $(45 \mathrm{~g} / \mathrm{L}$; RR 55 to $73 \mathrm{~g} / \mathrm{L})$. The total nucleated cell counts ranged from 1300 to $12800 \times 10^{6} / \mathrm{L}$ (median $3000 \times 10^{6} / \mathrm{L}$ ) with a mixture of non-degenerate neutrophils and macrophage/monocyte/mesothelial cells consistent with modified transudates or non-septic exudates.

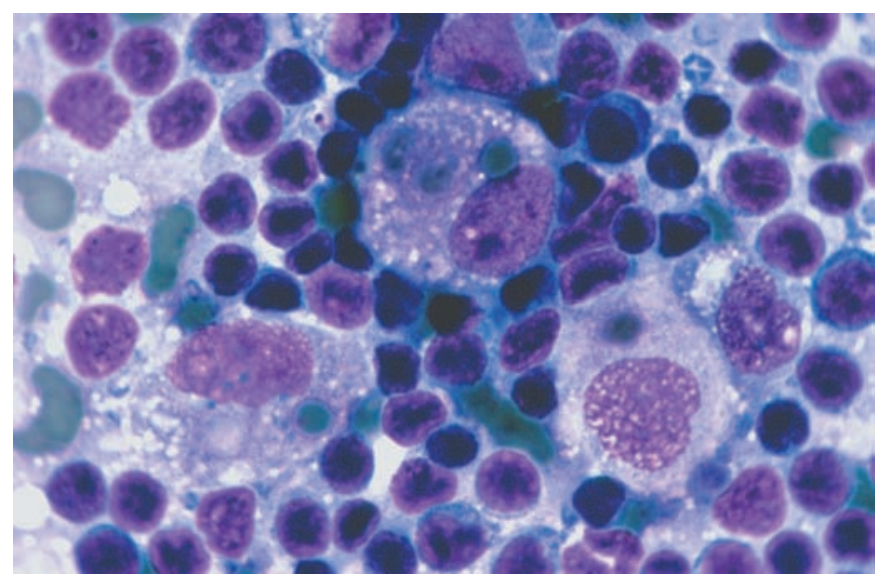

Figure 2. Biopsy of a mesenteric lymph node from a 1-year-old Burmese cat diagnosed with feline infectious peritonitis. Note the three macrophages in the centre with intact erythrocytes in their cytoplasm, depicting erythrophagocytosis. Haematoxylin and eosin (x 330 magnification). Photo courtesy of Dr Patricia Martin.

\section{Cytology from ultrasound-guided aspirates}

Ultrasonography was used to confirm or establish the presence of abdominal organomegaly in 12 cats and to guide the site of aspiration. Aspirates were taken from the mesenteric lymph nodes of five cats, of which four were deemed to have reactive lymph nodes, while one had evidence of pyogranulomatous inflammation. Liver aspirates from two cats did not contribute to the diagnosis. Aspirates were taken from kidney lesions in three cats, of which two showed evidence of pyogranulomatous inflammation, while one was non-diagnostic. An aspirate from a thickened colonic wall in one cat revealed pyogranulomatous inflammation. An aspirate from a splenic lesion in one cat revealed lymphoid hyperplasia. Overall, in the 12 cats in which ultrasound guided aspirates were performed, five were determined to have pyogranulomatous inflammation present in the aspirated organ. 
Table 3. Results of abdominal or thoracic fluid analysis in 13 cats with effusive feline infectious peritonitis.

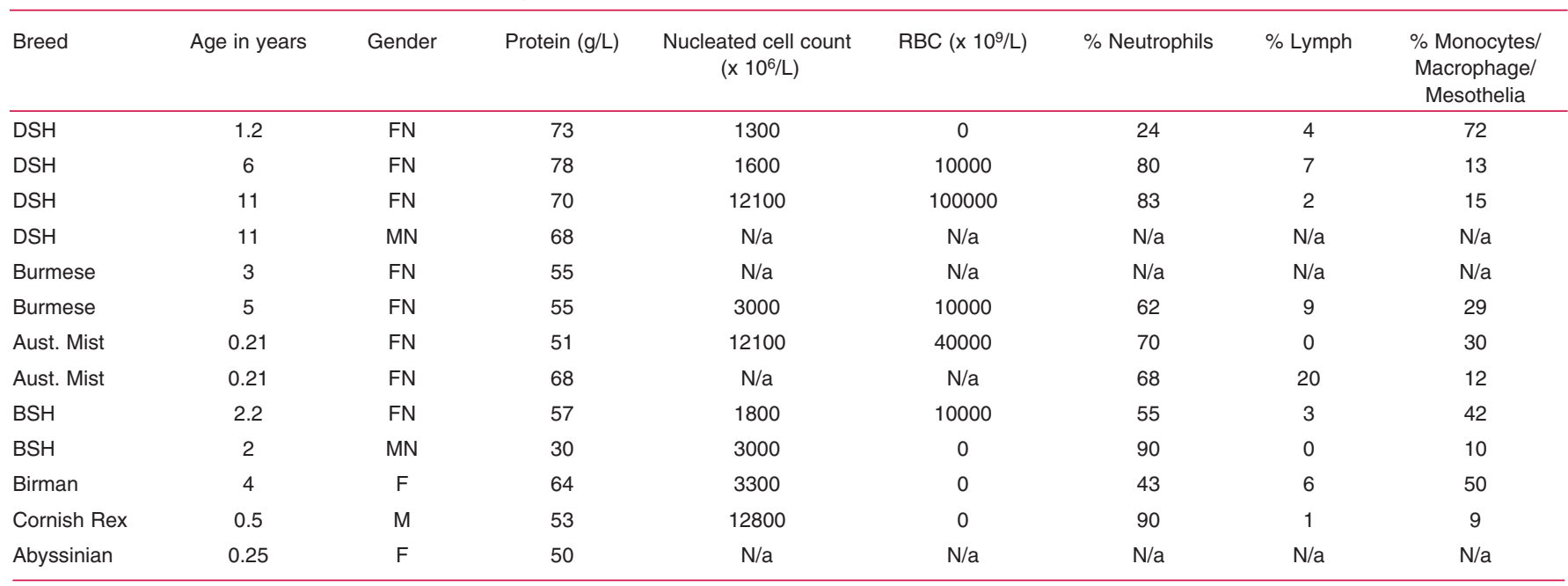

$\mathrm{DSH}=$ domestic shorthair; $\mathrm{BSH}=$ British shorthair; $\mathrm{RBC}=$ red blood cells; Lymph = Lymphocytes; FN = female neuter; $\mathrm{MN}=$ male neuter; $\mathrm{M}=$ male; $\mathrm{F}=$ female; $\mathrm{N} / \mathrm{a}=$ not available

Table 4: Cerebrospinal fluid analysis in four cats diagnosed with feline infectious peritonitis.

\begin{tabular}{|c|c|c|c|c|c|c|c|}
\hline Breed & Age (years) & CSF protein (g/L) & Nucleated cell count $(/ \mu \mathrm{L})$ & $\mathrm{RBC}(/ \mu \mathrm{L})$ & $\%$ Neut & $\%$ Lymp & $\%$ Mono \\
\hline DSH & 0.33 & 0.25 & 1200 & 0 & 92 & 3 & 5 \\
\hline DSH & 4 & 0.77 & 434 & 17 & 70 & 15 & 15 \\
\hline Burmese & 0.58 & $\mathrm{~N} / \mathrm{a}$ & 547 & 1573 & 14 & 72 & 14 \\
\hline Burmese & 11 & 1.3 & 1040 & 19 & 80 & 17 & 3 \\
\hline
\end{tabular}

Neut $=$ Neutrophil Lymp = lymphocyte Mono = Monocytes $;$ CSF $=$ cerebrospinal fluid $;$ RBC $=$ red blood cell .

Normal ranges derived from Rand et al; 37 CSF protein < $0.3 \mathrm{~g} / \mathrm{L} ;$ Nucleated cell count $<2$ per $\mathrm{mL} ; \mathrm{RBC}<30$ per $\mathrm{mL}$; Neutrophils $<9 \%$; Lymphocytes < $27 \%$; Monocytes $69-100 \%$.

CSF analysis - Cerebrospinal fluid analysis was attempted in five of ten cats that showed clinical signs of neurological disease. One of the five cats died during attempted cisternal CSF collection. The results of CSF analysis on the remaining four cats are listed in Table 4. The CSF total protein was elevated in two of three cats in which sufficient CSF was collected and ranged from 0.77 to 1.3 $\mathrm{g} / \mathrm{L}(\mathrm{RR}<0.25 \mathrm{~g} / \mathrm{L})$. A neutrophilic pleocytosis was present in three of four cats in which a nucleated cell differential could be performed. In one cat lymphocytes were the predominant cell type. In this cat, some blood contamination of the CSF was present, but even if corrected for at the rate of one white blood cell per $500 \mathrm{RBC}$ per $\mathrm{mL}$, the level of pleocytosis was still marked. Histopathological examination of the CNS taken at necropsy in this cat showed a pyogranulomatous meningoencephalitis particularly affecting the choroid plexus and periventricular grey matter, with lesions most severe in the cerebellum and adjacent brain stem. Mild hydrocephalus was also present.

Histopathology — In all cats in this study, the diagnosis of FIP was confirmed by histopathological examination of tissues. Histopathology was typical of FIP in the following tissues/body system: liver (64\% of cats), abdominal lymph nodes $(60 \%)$, gastrointestinal tract $(55 \%)$, kidney $(38 \%)$, spleen $(36 \%)$, neurological system $(29 \%)$, lung $(24 \%)$, pancreas $(21 \%)$, eyes $(12 \%)$, mesentery (12\%), omentum (5\%) and heart (5\%). Full necropsy examinations were performed in 37 cats, while FIP was diagnosed in the other five cats following histopathological examination of biopsy material from one or more tissues.
Two cats had non-effusive, localised ileocaecocolic FIP with lesions also present in mesenteric lymph nodes. While clinical signs in both cases were confined to the gastrointestinal tract, neither cat received a full necropsy to exclude the presence of lesions elsewhere in the body.

Four cats had localised CNS disease.

Unfortunately, histopathological examination was not conducted on the enlarged mandibular lymph nodes in any of the cats and therefore the aetiology of the lymphadenomegaly remains unknown.

Correlation between histopathology, clinical signs and clinical pathology Of the 27 cats with histological lesions in the liver consistent with FIP, blood biochemical analyses were performed in 23, of which only three had elevations of ALT, and a further three cats had elevations of AST in the absence of ALT or CK elevations. Eleven of the 27 cats had jaundice and hyperbilirubinaemia.

Of the 16 cats with histological lesions suggestive of FIP in one or both kidneys, none were azotaemic, two were polyuric/polydipsic, while three had palpable renomegaly.

Of the 23 cats with gastrointestinal lesions seen histologically, 15 had clinical signs of vomiting and/or diarrhoea, as well as palpable enlargement of some portion of the GIT.

Of the 12 cats with histological CNS lesions, six had neurological disease combined with disease in other organs, four had neurological disease alone, while two did not have any neurological signs prior to euthanasia. 
Figure 3. Cross-section of left and right kidneys from a cat with histological lesions consistent with FIP. Azotaemia was not present and urine concentration exceeded 1.040 .

\section{Discussion}

The clinical similarity between FIP and many other feline diseases, coupled with the non-specific nature of many of the tests available, makes the definitive diagnosis of FIP difficult even when the diagnostic approach is broad and thorough. The advent of serological tests and RT-PCR has added to this complexity due to the indistinct differentiation between FECV and FIPV, limiting the specificity of these tests. While a cat presented with hyperproteinaemia, fever, and high protein ascites and/or pleural effusion may be highly suggestive of FIP, the variation in clinical manifestations beyond this stereotype is remarkable. This study illustrates the extreme variability in clinical presentations of FIP and represents the first peer-reviewed article characterising FIP in Sydney, Australia since the first case reports in 1974. 4,5 Significant features of this study that are unique to the contemporary literature are i) the over-representation of certain breeds (Burmese, Australian Mist, BSH, and Cornish Rex) and the under-representation of other breeds (DSH, Persian); ii) the overrepresentation of males; iii) the tendency for effusive disease in Australian Mist cats and non-effusive disease in Burmese; iv) the even age distribution of disease seen in cats older than 2 years-of-age; and v) the presence of fulminant immune-mediated haemolytic anaemia in two cats.

FIP is reported to occur more frequently in cats from catteries, boarding facilities and multicat households. ${ }^{15}$ While no breed predisposition has been firmly established, certain bloodlines and matings within a breed may be at greater risk. ${ }^{8}$ The incidence of FIP in purebred catteries increases in proportion to the number of animals kept in that facility ${ }^{16}$ and is facilitated by the faeco-oral transmission of FCoV and the persistence of the virus within the environment of closely confined animals. Environmental factors, however, are not the sole contributor to FIP susceptibility. Foley and Pedersen ${ }^{17}$ determined that FIP susceptibility was a partially heritable trait regulated by multiple loci, with close relatives of affected cats within four Persian and one Birman catteries being significantly more likely to die of FIP than unrelated cats. ${ }^{17}$ Of the non-domestic felids, cheetahs have been found to be especially susceptibility to FIP. Inbreeding in this species has led to low major histocompatibility complex polymorphism, predisposing them to diseases involving a variety of pathogens, including FIP. ${ }^{18}$ Conversely, cats from Abyssinian bloodlines have been reported to have significantly longer survival times after challenge with virulent FIP strains than other breeds, suggesting an inherited resistance to $\mathrm{FIP}^{17,19}$

In the current study, purebred cats were over represented in the FIP cohort while DSH were under represented. Within the purebred cats, the Burmese, Australian Mist, BSH and Cornish Rex cats were significantly over-represented, while Persian cats were under-represented, dispelling the explanation of husbandry practices being the major contributor to the incidence of FIP. Two of the affected Australian Mist cats were siblings but unfortunately the genetic relationships between affected cats within other breeds were not available. Further examination of the pedigree data from numerous generations of cats within these breeds is required to determine the nature of their susceptibility to FIP. Preliminary studies by Addie and colleagues ${ }^{20}$ investigating the relationship of feline leucocyte antigen class II polymorphism and susceptibility to FIP failed to link any particular alleles with the development of FIP, carrier status, transient infection or resistance. On the other hand, Burmese cats in that study were found to have fewer alleles than other breeds, which may suggest reduced major histocompatibility polymorphism. The genetic similarity between Burmese in the UK and Australia makes this research area relevant to the current study and further studies involving both genetic pools would be useful.

Whilst the majority of reports worldwide have found no gender predisposition for FIP, ${ }^{8}$ others have suggested that the disease is more prevalent in males. ${ }^{21-23,38}$ In the current study an overrepresentation of males was found, and while the numbers were too small to permit statistical analysis within each breed, the ratio of males to females of 7:3 in Burmese cats was higher overall than in the FIP cohort. Subsequent to this study, cases of FIP presented to the UVCS either as patients or diagnostic specimens has seen a continuation of this male over-representation, most notably in Burmese and BSH cats. Further studies into potential differences in the host immune response to FIPV within these breeds are essential.

Approximately half of the cats with FIP are under 2-years-old in surveys worldwide. 8,15 This is consistent with the current study in which $55 \%$ of cats were under 2 years and no relationship was found between the type of disease seen and the age of the cat. Earlier papers ${ }^{24,25}$ reported a bimodal distribution of cats affected with FIP, with the highest incidence occurring between 6 months and 5 years, a decline between 5 and 13 years, followed by an increased incidence in cats 14 to 15 years. In the current study the oldest cat in which FIP was diagnosed was 11 -years-old and there was an even distribution of affected cats between the ages of 2 and 11 , illustrating that no cat is beyond suspicion.

Haematological and biochemical findings reported in association with FIP are non-specific and include hyperproteinaemia due to hyperglobulinaemia, hyperbilirubinaemia, normocytic normochromic anaemia, and leukocyte changes traditionally seen with stress (leukocytosis associated with a neutrophilia, lymphopenia and eosinopenia). Leukopenia has also been reported occasionally. In addition, analytes specific to affected organs may be elevated. In the current study total plasma protein was elevated above 78 $\mathrm{g} / \mathrm{L}$ in $60 \%$ of cats tested and hyperproteinaemia was seen with equal frequency in effusive and non-effusive FIP. Pedersen ${ }^{24}$ found an elevation of total plasma protein above $78 \mathrm{~g} / \mathrm{L}$ in $55 \%$ of effusive FIP and $71 \%$ of non-effusive FIP cases, while Sparkes and colleagues ${ }^{26}$ have reported hyperproteinaemia in as few as $39 \%$ of cats with confirmed FIP, with a similar frequency seen in effusive and non-effusive cases. Hartman and colleagues, ${ }^{27}$ in the largest study of FIP cases to date, compared the value of certain diagnostic tests and found that elevations of total serum proteins above $80 \mathrm{~g} / \mathrm{L}$ had a specificity of 0.60 and sensitivity of 0.62 . This highlights the need to view hyperproteinaemia as being supportive of a diagnosis of FIP and several other diseases, but of 
limited diagnostic value when in the normal range. In cases in which total serum protein is elevated, additional supportive evidence for FIP can be gleaned from measurement of albuminglobulin ratios, $\gamma$-globulin concentrations and $\alpha 1$-acid glycoprotein.

Non-regenerative anaemia, a commonly reported finding in many chronic feline diseases, was found in $60 \%$ of cats in the current study. The cause of non-regenerative anaemia in FIP is frequently not identified and is attributed to chronic inflammation. ${ }^{15}$ In the current study, two cats developed fulminant haemolytic anaemia that was refractory to blood transfusion and/or broad-spectrum antibiotics, including doxycycline. Corticosteroids were not used. Subsequent to this study a further two cats diagnosed with FIP by Veterinary Pathology Diagnostic Services, The University of Sydney, have similarly died of fulminant haemolytic anaemia. This is the first report to the authors' knowledge of fulminant refractory haemolytic anaemia in cats with confirmed FIP. In all four cases, haemolysis was aggressive and fatal and was considered most likely secondary immune-mediated in origin. Investigation for specific precipitating causes such as FeLV or the haemotrophic Mycoplasma species was not performed in these cats so the potential role of these agents cannot be excluded.

The lack of correlation in many cases between the presence of histological lesions of FIP and supporting clinicopathological evidence for disease was especially noteworthy when lesions were present in the liver or kidneys. Some reports have suggested that involvement of the kidneys in this disease process leads to azotaemia and electrolyte disturbances, ${ }^{28}$ while others discuss the variable increases in measurements depending on the extent and location of disease. ${ }^{27}$ In the current study none of the 16 cats with renal involvement had azotaemia, illustrating the insensitivity of these tests in determining renal disease in general, despite the extent of pathology in some cases (Figure 3). In cats with liver involvement $11 / 27$ had hyperbilirubinaemia and jaundice, but very few had elevations of ALT or AST to indicate the involvement of this organ in the disease process. While haematological and serum biochemical profiles can provide essential baseline information in the investigation of sick cats, clinicians cannot exclude disease in certain body systems simply on the absence of abnormal findings.

The frequency with which effusive FIP is seen in relation to noneffusive disease varies widely. In a study of 488 cats with histopathologically confirmed FIP, ${ }^{27}$ it was found that $80 \%$ of cats had effusive disease compared with $60 \%$ of cats in studies by Lutz and colleagues. ${ }^{29}$ The current study had approximately equal numbers of cats with effusive (45\%) and non-effusive (55\%) disease. While it may be argued that this was the result of many of the cases being derived from a referral hospital, $\mathrm{PCH}$ (a predominantly primary accession hospital) had $60 \%$ non-effusive cases. Although the number of cases is small, these results would suggest that effusive disease is not seen as frequently in Australia.

The analysis of pleural and peritoneal effusions is an essential diagnostic test in effusive FIP and the findings of this study were similar to previous reports. ${ }^{24,30,31}$ Protein electrophoresis has been reported as a useful addition to fluid analysis, with albumin to globulin ratios greater than 0.81 being highly predictive for excluding FIP from the differential diagnosis. ${ }^{30}$ Protein electrophoresis was not performed in any of the cats in this study. Sparkes and colleagues ${ }^{31}$ found that all 16 cats with FIP had fluid protein concentrations greater than $35 \mathrm{~g} / \mathrm{L}$, with globulins constituting greater than $50 \%$ of the proteins. These two findings together were found to have specificity for FIP of 0.90 . The single cat in the present study with an effusion protein concentration below $35 \mathrm{~g} / \mathrm{L}$ also had concurrent hypoproteinaemia associated with a mild hypoalbulinaemia and normal globulin levels, which is likely to have influenced this result.

Cytologically, FIP effusion typically consists primarily of nondegenerate neutrophils mixed with mesothelial/macrophage-type cells, with nucleated cell counts generally 2000 to $6000 \times 10^{6} / \mathrm{L}$, although cell counts 500 to $25000 \times 10^{6} / \mathrm{L}$ have been reported. ${ }^{28}$ In acute cases, a high ratio of neutrophils to mononuclear cells is found, whereas chronicity leads to a greater proportion of macrophages/mesothelial type cells. ${ }^{28}$ The presence of a high protein effusion and low to moderate cellularity, as described above, is helpful in providing further evidence for the likelihood of FIP, but has been found in other disease processes such as renal carcinoma and lymphocytic cholangitis, in which it has been reported to induce effusions that may be indistinguishable from FIP grossly and cytologically. . $^{31,32}$

Both the value and pitfalls of ultrasound-guided fine needle aspiration of abdominal organs affected by FIP was evident from the cases presented here. Of the 12 cats in which sonography was performed, five were determined to have pyogranulomatous inflammation in the aspirated organ, which is suggestive rather than pathognomic for FIP, while seven were non-diagnostic. The use of cytology to support a diagnosis of FIP, even with the addition of immunocytochemical detection of $\mathrm{FCoV}$ positive macrophages, has been a low yield procedure (S Paltrinieri, personal communication). This reflects the variable and often sparse distribution of lesions throughout an affected organ as well as the limitations of sonography in locating these microscopic areas.

Neurological disease is a common clinical manifestation of FIP worldwide, occurring in 25 to $33 \%$ of cases and with a preponderance of cases having non-effusive disease. In the present study, 12 cats $(29 \%)$ had histological lesions suggestive of FIP meningoencephalitis, with only one cat having effusive disease and 10 (23\%) presenting with clinical signs of neurological disease. Confirming an antemortem diagnosis of FIP is an especially difficult task if neurological disease is present alone. Rand and colleagues $^{33}$ have suggested that FIP can be differentiated from other causes of CNS disease when CSF protein level is greater than $2 \mathrm{~g} / \mathrm{L}$ and cytological analysis shows $>100$ nucleated cells $/ \mathrm{mL}$, comprising $>70 \%$ neutrophils. In the current study, despite histopathological evidence of neurological FIP at necropsy, only one of four cats fulfilled the above criteria, although all cats had nucleated cell counts considerably greater than 100 cells $/ \mu \mathrm{L}$. A neutrophilic pleocytosis was present in three of the four cats. Determining the antibody titre to FCoV in CSF compared to serum is still considered to be helpful in establishing a diagnosis of FIP, but was not performed in these cases. It is widely known that collection of CSF is certainly not a risk free procedure, as evidenced by one of the five cats dying as a result of cisternal CSF collection. Without a definitive diagnostic test, clinicians must determine the risks and benefits of this procedure on a case-by-case basis. Specialist imaging using computed tomography or magnetic resonance imaging was not performed on any of the patients, despite recent work showing periventricular changes in affected cats.

The difficulties of interpreting serological tests for FCoV have led to widespread acceptance that diagnosis of FIP by serological means alone is neither possible nor appropriate. ${ }^{8,10,34,35}$ Despite 
this, serology continues to be given undue emphasis in the diagnosis of FIP. The antibody titre to FCoV in cats with FIP varies according to the nature of the clinical syndrome. There is considerable variation in the reported magnitude of anti FCoV antibody titres in cats with confirmed effusive or non-effusive FIP, with considerable overlap with healthy patients, making interpretation difficult. ${ }^{35,36}$ Indeed, a negative antibody titre alone cannot rule out a diagnosis of FIP, while a positive titre does not confirm a diagnosis of FIP. The emergence of serological tests that claim to measure antibodies against FIPV alone have become popular but their validity is not supported by peer reviewed publications and currently they are considered of no greater value than other serological tests. ${ }^{10}$ In the current study, serological tests were performed in very few cats, but the results exemplify the difficulties and dangers of interpretation.

A study looking at the seroprevalence of $\mathrm{FCoV}$ in different subsets of Australian cats (for example feral versus household, pedigree versus domestic, male versus female, free range versus indoors) is required before we can interpret serology on sick cats or consider institution of control programs in catteries. This study is currently underway at The University of Sydney and forms the basis of other papers in this series on FIP and FCoV. Knowledge of the prevalence of $\mathrm{FCoV}$ in Australia, especially in our breeding catteries, is an important first step in understanding the epidemiology of FIP in Australia and may form the basis of a control program for this virus, especially with breeds such as the Australian Mist, Burmese, BSH and Cornish Rex. In addition, the development of immunocytochemistry and immunohistochemistry to supplement routine fluid analysis and histopathology, currently underway in our laboratory, is necessary to provide a more accurate and in the case of immunocytochemistry, less invasive, means of confirming the diagnosis in the future.

\section{Acknowledgments}

The authors are thankful for the diligence and foresight of Trish Martin who captured the cytological images from two of these cases numerous years ago and made them available for this journal article. The authors are very grateful to the clinicians, Sue Foster, Julia Beatty, Vanessa Barrs, Carolyn O’Brien, Elizabeth DillMacky, Barbara Chapman, David Church and Jill Maddison, who also diagnosed and treated some of these cats and whose case records form the basis of this article. Jacqui Norris and Richard Malik are forever grateful for the guidance and support given to them by the late Professor Daria Love.

\section{References}

1. Pedersen NC, Floyd K. Experimental studies with three new strains of feline infectious peritonitis virus: FIPV-UCD2, FIPV-UCD3, and FIPV-UCD4. Compend Contin Educ Prac Vet 1985;7:1001-1011.

2. Poland AM, Vennema H, Foley JE, Pedersen NC. Two related strains of feline infectious peritonitis virus isolated from immunocompromised cats infected with a feline enteric coronavirus. J Clin Microbiol 1996;34:3180-3184.

3. Holzworth J. Some important disorders of cats. Cornell Vet 1963;53:157-160. 4. Jones BR, Hogg GG. Feline infectious peritonitis. Aust Vet J 1974;50:398-402.

5. Watson ADJ, Huxtable CRR, Bennett AM. Feline infectious peritonitis. Aust Vet J 1974;50:393-397.

6. Pedersen NC, Boyle JF, Floyd K. Infection studies in kittens, using feline infectious peritonitis virus propagated in cell culture. Am J Vet Res 1981:42:363-367.

7. Vennema HP, Poland A, Hawkins KF, Pedersen NC. A comparison of the genomes of FECVs and FIPVs and what they tell us about the relationships between feline coronaviruses and their evolution. Feline Pract 1995;23:40-44.
8. Pedersen NC. An overview of feline enteric coronavirus and infectious peritonitis virus infections. Feline Pract 1995;23:7-20.

9. Pedersen NC. Feline infectious peritonitis and feline enteric coronavirus infections. 2. Feline infectious peritonitis. Feline Pract 1983;13:5-20.

10. Addie DD, Paltrinieri S, Pedersen NC. Recommendation from workshops of the second international feline coronavirus/feline infectious peritonitis symposium. $J$ Fel Med Surg 2004:6:125-130.

11. Herrewegh AAPM, Egberink HF, Horzinek MC, Rottier PJM, DeGroot RJ. Polymerase chain reaction (PCR) for the diagnosis of naturally occurring feline coronavirus infections. Feline Pract 1995;23:56-60.

12. Gamble DA, Lobbiani A, Gramegna M, Moore LE, Colucci G. Development of a nested PCR assay for detection of feline infectious peritonitis virus in clinical specimens. J Clin Microbiol 1997;35:673-675.

13. Simons FA, Rottier PJM, Rofina $\mathrm{J}$ et al. Detection of replicating Feline Coronavirus in peripheral blood mononuclear cells as a potential diagnostic assay for Feline Infectious Peritonitis. Second International Feline Coronavirus/Feline Infectious Peritonitis Symposium, Glasgow, Scotland, 2002.

14. Barlough JE, Stoddart CA. Feline infectious peritonitis. Vet Rep 1988;1:1,4-5. 15. Addie D, Jarrett O. Feline coronavirus infection. In: Greene CE, editor. Infectious Diseases of the Dog and Cat. 2nd edn. Saunders, Philadelphia, 1998:58-68.

16. Kass $\mathrm{PH}$, Dent TH. The epidemiology of feline infectious peritonitis in catteries. Feline Pract 1995;23:27-32.

17. Foley JE, Pedersen NC. The inheritance of susceptibility to feline infectious peritonitis in purebred catteries. Feline Pract 1996;24:14-22.

18. O'Brien JJ, Roelke ME, Marker L. Genetic basis for species vulnerability in the Cheetah. Science 1985;227:1428-1434.

19. Vennema HP, DeGroot RJ, Harbour DA. Primary structure of the membrane and nucleocapsid protein genes of feline infectious peritonitis virus and immunogenicity of recombinant vaccinia viruses in kittens. Virology 1991;181:327-335.

20. Addie DD, Kennedy LJ, Ryvar R et al. Feline leukocyte antigen class II polymorphism and susceptibility to feline infectious peritonitis. J Fel Med Surg 2004;6:59-62.

21. Robinson RL, Holzworth J, Gilmore CE. Naturally occurring infectious peritonitis: signs and clinical diagnosis. J Am Vet Med Assoc 1971;158:981-986.

22. Potkay A, Bacher JD, Pitts TW. Feline infectious peritonitis in a closed breeding colony. Lab Anim Sci 1974;24:279-289.

23. Schalm OW. Feline infectious peritonitis: vital statistics and laboratory findings. Feline Pract 1973;3:5, 18-20.

24. Pedersen NC. Feline infectious peritonitis: something old, something new. Feline Pract 1976;6:42-46, 48-51.

25. Pedersen NC. Feline infectious peritonitis and feline enteric coronavirus infections. I. Feline enteric coronaviruses. Feline Pract 1983;13:5-20.

26. Sparkes AH, Gruffydd-Jones TJ, Harbour DA. Feline infectious peritonitis: a review of clinicopathological changes in 65 cases, and a critical assessment of their diagnostic value. Vet Rec 1991;129:209-212.

27. Hartmann $\mathrm{K}$, Binder $\mathrm{C}$, Hirschberger $\mathrm{J}$ et al. Comparison of different tests to diagnose feline infectious peritonitis. J Vet Intern Med 2003;17:781-790.

28. Weiss RC, Scott FW. Laboratory diagnosis of feline infectious peritonitis. Feline Pract 1980;10:16-22.

29. Lutz H, Hauser B, Horzinck MC. Feline infectious peritonitis (FIP)-the present state of knowledge. J Small Anim Pract 1986;27:108-116.

30. Shelly SM, Scarlett-Kranz J, Blue JT. Protein electrophoresis on effusions from cats as a diagnostic test for feline infectious peritonitis. J Am Anim Hosp Assoc 1988;24:495-500.

31. Sparkes AH, Gruffydd-Jones TJ, Harbour DA. An appraisal of the value of laboratory tests in the diagnosis of feline infectious peritonitis. J Am Anim Hosp Assoc 1994;30:345-350.

32. Lucke VM, Davis JD. Progressive lymphocytic cholangitis in the cat. J Small Anim Pract 1984;25:249-260.

33. Rand JS, Parent J, Percy D, Jacobs R. Clinical, cerebrospinal fluid, and histological data from twenty-seven cats with primary inflammatory disease of the central nervous system. Can Vet J 1994;35:103-110.

34. Addie DD, Jarrett JO. Feline coronavirus antibodies in cats. Vet Rec 1992;131:202-203.

35. Sparkes AH, Gruffydd-Jones TJ, Harbour DA. Feline coronavirus antibodies in UK cats. Vet Rec 1992;131:223-224.

36. Sparkes AH, Gruffydd-Jones TJ, Harbour DA, Hopper CD, Smerdon TN Blood tests for FIP. Vet Rec 1990;126:225.

37. Rand JS, Parent J, Jacobs R, Percy D. Reference intervals for feline cerebrospinal fluid: cell counts and cytologic features. Am J Vet Res 1990;51:10441048.

38. Rohrbach BW, Baldwin CA, Reed WM. Epidemiology of feline infectious peritonitis among cats examined at veterinary medical teaching hospitals. J Am Vet Med Assoc 2001;218:1111-1115.

(Accepted for publication 18 March 2005) 